\title{
Prognostic significance of preoperative neutrophil-to-lymphocyte ratio in papillary renal cell carcinoma patients after receiving curative surgery based on a retrospective cohort
}

Zhilei Zhang ${ }^{1,2}$, Yongbo Yu ${ }^{1,2}$, Jilu Zheng ${ }^{1,2}$, Mingxin Zhang ${ }^{1}$ and Haitao Niu ${ }^{\text {* }}$

\begin{abstract}
Background: Inflammatory response biomarkers have been studied as promising prognostic factors in renal cell carcinoma, but few studies have focused on papillary renal cell carcinoma (PRCC). This study was performed to evaluate the prognostic value of the preoperative neutrophil-to-lymphocyte ratio (NLR) in PRCC patients.

Methods: In total, 122 postoperative PRCC patients selected from 366 non-clear cell renal cell carcinoma patients were enrolled from our institution between 2012 and 2020. The optimal cutoff value of the NLR was assessed by receiver operating characteristic (ROC) curve analysis, and the Kaplan-Meier method and Cox's proportional hazards regression models were performed to analyze the association of the NLR with overall survival (OS). In addition, the potential of tumor-node-metastasis (TNM) stage, the NLR and an NLR-TNM system to predict survival were compared with ROC curves, and clinical usefulness of the predicting models were assessed by decision curve analysis.

Results: A threshold value of 2.39 for the NLR for OS analysis was determined by ROC curve analysis. An NLR $\geq 2.39$ was associated with a more advanced TNM stage $(P<0.01)$ and larger tumors $(P<0.05)$ than a low NLR, as well as pathological subtype II $(P<0.05)$, and the patients with a high NLR also exhibited significantly worse overall survival outcomes $(P<0.05)$. The NLR was determined to be a significant independent prognostic indicator by univariable and multivariable analyses ( $H R=5.56, P<0.05)$. Furthermore, TNM stage and the NLR were integrated, and the area under the curve (AUC) of for the NLR-TNM system was larger than that of for the TNM system when predicting overall survival $(0.84$ vs $0.73, P=0.04)$. Decision curve analysis also demonstrated a better clinical value for the NLR-TNM model to predict the prognosis.
\end{abstract}

Conclusion: A high preoperative NLR was associated with poor clinical and pathologic parameters in patients with PRCC; moreover, the NLR was also an independent prognostic factor for the OS of patients with PRCC. The NLR-TNM system, which was a model that integrated the NLR with TNM staging, could improve the ability to predict overall survival.

Keywords: Papillary renal cell carcinoma (PRCC), Neutrophil-to-lymphocyte ratio (NLR), Overall survival (OS)

*Correspondence: niuht0532@126.com

${ }^{1}$ Department of Urology, The Affiliated Hospital of Qingdao University, Qingdao, China

Full list of author information is available at the end of the article

\section{Background}

Renal cell carcinoma (RCC) is a common malignant tumor of the urinary system. In 2018, approximately 
175,000 RCC patients died worldwide, and approximately 400,000 new cases were diagnosed [1]. Widespread use of abdominal ultrasound and computed tomography (CT) has increased the number of incidentally detected RCCs [2]. Histologically, RCC can be divided into clear cell renal cell carcinoma (ccRCC), and non-clear cell carcinoma. Papillary renal cell carcinoma (PRCC) is a common subtype of non-clear cell carcinoma, accounting for $15-20 \%$ of RCC [3]. The 5-year overall survival rate of PRCC is reported to be significantly higher than that of clear cell carcinoma (80.5\% vs $71.3 \%$ ) [4]. Surgery is still the main treatment of PRCC, and recurrence after surgery often occurs in the advanced stage of PRCC, which may threaten survival and quality of life to a certain extent. Substantial evidence shows that inflammatory cells can induce tumor cell proliferation and metastasis through systemic inflammatory activation; therefore, inflammatory cells may be used to predict tumor prognosis [5-7]. Several indicators of systemic inflammation, such as c-reactive protein (CRP), the neutrophil-to-lymphocyte count ratio (NLR) and the platelet-to-lymphocyte count ratio (PLR), have been studied to predict the prognosis of various tumors $[8,9]$. Among these indicators, the NLR is considered a valuable indicator for predicting tumor prognosis, such as that of lung cancer, liver cancer, esophageal cancer and ovarian cancer [10-12]. At present, there are few studies focusing on the prognosis of renal papillary cell carcinoma domestically or globally. Therefore, we used this background to analyze the value of the NLR in evaluating the prognosis of patients with renal papillary cell carcinoma following surgery.

\section{Methods}

\section{Patients}

A total of 366 patients with non-clear cell renal cell carcinoma (nccRCC) who underwent surgical treatment in the department of urology in our hospital from January 2012 to April 2020 were collected.

Inclusion criteria: (1) the medical records were complete. (2) No adjuvant treatment was performed before the operation. (3) The results of blood samplings were obtained within 1 week after admission. (4) The surgical method was partial nephrectomy or radical nephrectomy, and pathological results of specimens were confirmed by more than 2 pathologists as nccRCC with clean cutting edge and no residual.

Exclusion criteria: (1) patients with recent infectious diseases or a history of autoimmune diseases and infectious diseases; (2) patients with a history of primary tumor in other organs; (3) patients with preoperative radiotherapy or chemotherapy; (4) incomplete laboratory and pathological data.
After screening, a total of 122 patients diagnosed as papillary renal cell carcinoma (PRCC) were included in this study. This study was approved by the ethics committee of The Affiliated Hospital of Qingdao University.

\section{Data collection}

During the first week after admission, blood samples were obtained from all the patients. According to the results of peripheral blood test, neutrophil count $\left(\times 10^{9} / \mathrm{L}\right) /$ lymphocyte count $\left(\times 10^{9} / \mathrm{L}\right)$ was used to calculate the value of NLR. The optimal cutoff value of NLR was identified by an ROC analysis with OS as the primary endpoint, 2.39 was defined as the best cutoff value due to the maximum youden index value (sensitivity: $78.6 \%$; specificity: $70.4 \%$; youden index: 0.49 ). The cutoff value of CHOL (4.90) were defined by ROC with OS as the endpoint as well.

Follow up strategy: All patients after surgery were considered to be healthy, the first follow-up time in the first 2 years after the operation was every 3 months after operation, for the next 2 years, the follow up time was every 6 months and then annually for the last years, the followup included medical history, physical examination, laboratory blood tests and image information, information of death obtained by telephone interview was recorded. The follow-up period was up to 2020-11-01.

\section{Statistics}

Association with NLR between groups was analyzed by Chi-square test and Mann-Whitney $U$ test, and the optimal threshold value of NLR was determined by the receiver operating characteristic (ROC) curve with OS as the primary endpoint. Kaplan-Meier method was utilized to estimate the survival rate of OS, and the survival estimate between groups divided by each cutoff value was compared by log-rank test. Univariable and multivariable COX regression analysis were used to determine the independent prognostic factors of OS. The abilities of different factors to predict OS were compared by the comparisons of the area under curve (AUC) of each parameter, and decision curve analysis was used to assess the clinical usefulness of the predicting models. SPSS ver. 22.0 (SPSS Inc., Chicago, IL, USA), R 4.0.3 (formerly AT\&T, now Lucent Technologies), Graphpad Prism 8 (GraphPad Software Inc., La Jolla, CA, USA) and Medcalc v19.1.3 were used to analyze all the statistics. $P<0.05$ of two sides was considered statistically significant.

\section{Results}

\section{Patient characteristics}

The patient clinicopathological characteristics were listed on Table 1, 122 PRCC patients with 88 (72.1\%) males and 34 (27.9\%) females aged from 7 to 81 (the 
Table 1 Patient clinical characteristics

\begin{tabular}{|c|c|}
\hline Characteristic & No. of patients (\%) \\
\hline \multicolumn{2}{|l|}{ Age/years } \\
\hline$<60$ & $61(50 \%)$ \\
\hline$\geq 60$ & $61(50 \%)$ \\
\hline \multicolumn{2}{|l|}{ Gender } \\
\hline Male & $88(72.1 \%)$ \\
\hline Female & 34 (27.9\%) \\
\hline \multicolumn{2}{|l|}{ BMl } \\
\hline$\geq 25$ & $54(44.3 \%)$ \\
\hline$<25$ & $68(55.7 \%)$ \\
\hline \multicolumn{2}{|c|}{ Diabetes/hyperglycemia, n (\%) } \\
\hline Yes & $20(16.4 \%)$ \\
\hline No & $102(83.6 \%)$ \\
\hline \multicolumn{2}{|c|}{ TNM stage (AJCC8th) } \\
\hline 1 & $101(82.7 \%)$ \\
\hline$\|$ & $10(8.2 \%)$ \\
\hline III & $8(6.6 \%)$ \\
\hline IV & $3(2.5 \%)$ \\
\hline \multicolumn{2}{|l|}{ Surgical type } \\
\hline $\mathrm{RN}$ & $64(52.5 \%)$ \\
\hline PN & $58(47.5 \%)$ \\
\hline \multicolumn{2}{|l|}{ Tumor size $(\mathrm{cm})$} \\
\hline$<7$ & $98(80.3 \%)$ \\
\hline$\geq 7$ & $24(19.7 \%)$ \\
\hline \multicolumn{2}{|l|}{ Subtype, n (\%) } \\
\hline । & $64(55.6 \%)$ \\
\hline$\|$ & $58(44.4 \%)$ \\
\hline \multicolumn{2}{|c|}{ Serum album(g/l) } \\
\hline$\geq 40$ & $70(57.4 \%)$ \\
\hline$<40$ & $52(42.6 \%)$ \\
\hline \multicolumn{2}{|l|}{ NLR } \\
\hline$<2.39$ & 78 (63.9\%) \\
\hline$\geq 2.39$ & $44(36.1 \%)$ \\
\hline \multicolumn{2}{|l|}{$\mathrm{CHOL}$} \\
\hline$<4.90$ & $65(53.3 \%)$ \\
\hline$\geq 4.90$ & $57(46.7 \%)$ \\
\hline \multicolumn{2}{|l|}{$\mathrm{BUN} / \mathrm{Cr}$} \\
\hline$<20$ & $87(57.8 \%)$ \\
\hline$\geq 20$ & $35(42.2 \%)$ \\
\hline
\end{tabular}

TNM tumor-node-metastasis, $\mathrm{CHOL}$ cholesterol, NLR neutrophil-to-lymphocyte ratio, $P N$ partial nephrectomy, $R N$ radical nephrectomy

mean age was 57.7). According to the histology, these patients were divided into two subtypes 64 (52.5\%) I and 58 (47.5\%) II, respectively. All patients were underwent curative surgery including radical nephrectomy 64 (52\%) and partial nephrectomy 58 (47.5\%). Based on the 8th TNM classification of AJCC guideline, 101 (82.7\%), $10(8.2 \%), 8(6.6 \%)$ and $3(2.5 \%)$ were distributed into I, II, III and IV stage. The median follow up time was
33 months (interquartile range, 16.75-52.5 months), 14 patients were died during the follow-up, and the survival rate of 1 year, 3 years and 5 years were 99.2\%, $97.1 \%$ and $81.2 \%$ in our present study.

\section{Associations between NLR and clinicopathological characteristics of the patients}

ROC curve was used to identify the best cut-off value of NLR based on OS (overall survival) as the endpoint. The median preoperative NLR was 1.96 (IQR 1.42-2.82), and the optimal cut-off value of NLR was 2.39 in our study ( $83.3 \%$ sensitivity and $71.8 \%$ specificity) by ROC curves analysis, then 78 patients were categorized into the high NLR ( $\geq 2.39$ ) group and 44 patients were identified into the low NLR $(<2.39)$ group. Table 2 showed the association between different levels of NLR and clinicopathological characteristics, there were no significant differences between two groups with regard to the age, gender, BMI, surgical type and other clinical characteristics, patients with high NLR tend to have a more advanced TNM stage $(P<0.01)$ and larger tumors $(P<0.05)$ than a low NLR, as well as pathological subtype II $(P<0.05)$.

\section{Survival analysis}

The 1 year, 3 year and 5 year survival rates were $100 \%$, 98\%and 84\%in low NLR group, while the 1 year, 3 year and 5 year survival rates of high NLR group were $97.7 \%$, $80.6 \%$ and $56.1 \%$, respectively. The OS time of the high NLR group was shorter than the low NLR group $(P<0.01)$, Fig. 1 showed the Kaplan-Meier survival curves for OS of patients with PRCC according to NLR levels, TNM stage and Tumor size levels, which were all significant predictors for OS $(P<0.05)$ by the univariable analysis (Table 3), Patients with advanced TNM stage and larger tumor size were associated with worse OS.

In the univariable analysis the significant variables $(P<0.05)$ were included into the following multivariate analysis, as shown in Table 3, NLR (HR $=5.56,95 \%$ CI $1.06-29.15, P=0.04)$ and TNM stage $(\mathrm{HR}=4.86,95 \%$ CI 1.52-15.58, $P=0.01$ ) was the independent prognostic factors of OS, which demonstrated that patients with a NLR $\geq 2.39$ and advanced TNM stage were correlated with a shorter survival time.

\section{Comparison of the predictive prognostic value of TNM stage, NLR and NLR-TNM model}

To evaluate the ability of predicting OS in patients with PRCC, we further drew ROC curves for each parameter to calculated AUC of TNM stage, NLR and NLR-TNM (Fig. 2). The AUC of NLR-TNM system integrated NLR with TNM stage in predicting OS time were relatively higher than that of TNM and NLR alone ( 0.73 vs 0.78 vs 0.84 for TNM, NLR and NLR-TNM, respectively), 
Table 2 Associations between NLR and clinicopathologic characteristics

\begin{tabular}{|c|c|c|c|}
\hline Variables & $N L R<2.39(n=78)$ & $N L R \geq 2.39(n=44)$ & $P$ value \\
\hline Age/years & & & 0.451 \\
\hline$<60$ & $37(47.4 \%)$ & $24(54.5 \%)$ & \\
\hline$\geq 60$ & $41(52.6 \%)$ & $20(45.5 \%)$ & \\
\hline Gender & & & 0.596 \\
\hline Male & $55(70.5 \%)$ & $33(75 \%)$ & \\
\hline Female & $23(29.5 \%)$ & $11(25 \%)$ & \\
\hline $\mathrm{BMI}$ & & & 0.187 \\
\hline$\geq 25$ & $38(48.7 \%)$ & $16(36.4 \%)$ & \\
\hline$<25$ & $40(51.3 \%)$ & $28(63.6 \%)$ & \\
\hline Diabetes/hyperglycemia, n (\%) & & & 0.363 \\
\hline No & $67(85.9 \%)$ & $35(79.5 \%)$ & \\
\hline Yes & $11(14.1 \%)$ & $9(250.5 \%)$ & \\
\hline TNM stage (AJCC 8th) & & & $0.001^{\mathrm{a}}$ \\
\hline I & $71(91 \%)$ & $30(68.2 \%)$ & \\
\hline$\|$ & $4(5.1 \%)$ & $6(13.5 \%)$ & \\
\hline III & $3(3.9 \%)$ & $5(11.5 \%)$ & \\
\hline IV & 0 & $3(6.8 \%)$ & \\
\hline Surgical type & & & 0.063 \\
\hline $\mathrm{RN}$ & $36(46.2 \%)$ & $28(63.6 \%)$ & \\
\hline PN & $42(53.8 \%)$ & $16(36.4 \%)$ & \\
\hline Tumor size (cm) & & & 0.011 \\
\hline$<7$ & $68(87.2 \%)$ & $30(68.2 \%)$ & \\
\hline$\geq 7$ & $10(12.8 \%)$ & $14(31.8 \%)$ & \\
\hline Subtype, n (\%) & 0.022 & & \\
\hline 1 & $47(60.3 \%)$ & $17(38.6 \%)$ & \\
\hline$\|$ & 31 (39.7\%) & $27(61.4 \%)$ & \\
\hline Serum album (g/l) & & & 0.925 \\
\hline$\geq 40$ & 45 (57.7\%) & 25 (56.8\%) & \\
\hline$<40$ & $33(42.3 \%)$ & $19(43.2 \%)$ & \\
\hline $\mathrm{CHOL}$ & & & 0.085 \\
\hline$<4.90$ & $37(47.4 \%)$ & $28(63.6 \%)$ & \\
\hline$\geq 4.90$ & 41 (52.6\%) & $16(36.4 \%)$ & \\
\hline $\mathrm{BUN} / \mathrm{Cr}$ & & & 0.274 \\
\hline$<20$ & $53(67.9 \%)$ & $34(77.3 \%)$ & \\
\hline$\geq 20$ & 25 (32.1\%) & $10(22.7 \%)$ & \\
\hline
\end{tabular}

TNM tumor-node-metastasis, CHOL cholesterol, NLR neutrophil-to-lymphocyte ratio, $P N$ partial nephrectomy, $R N$ radical nephrectomy

a Mann-Whitney U

which revealed that the predictive ability of NLR was higher than traditional TNM stage, after integrated NLR with TNM stage system, the NLR-TNM showed the better ability in predicting OS time $(P<0.05)$ (Table 4).

Figure 3 showed the decision curves of the predicted models of NLR, TNM stage and NLR-TNM, and a positive net benefit was compared among them, which demonstrated that using this NLR-TNM model in the current study to predict OS added more benefits than the other two models.

\section{Discussion}

In our study, we respectively analyzed 122 PRCC patients who underwent curative surgical treatment. An NLR $\geq 2.39$ was closely correlated with a relatively advanced pathological stage and large tumor volume. In addition, the results demonstrated that a high preoperative NLR was an independent prognostic factor associated with shortened OS. These results were in line with other previous studies [13-15], which implies that the NLR is relevant for predicting poor clinical characteristics. Furthermore, the NLR-TNM system was relatively 


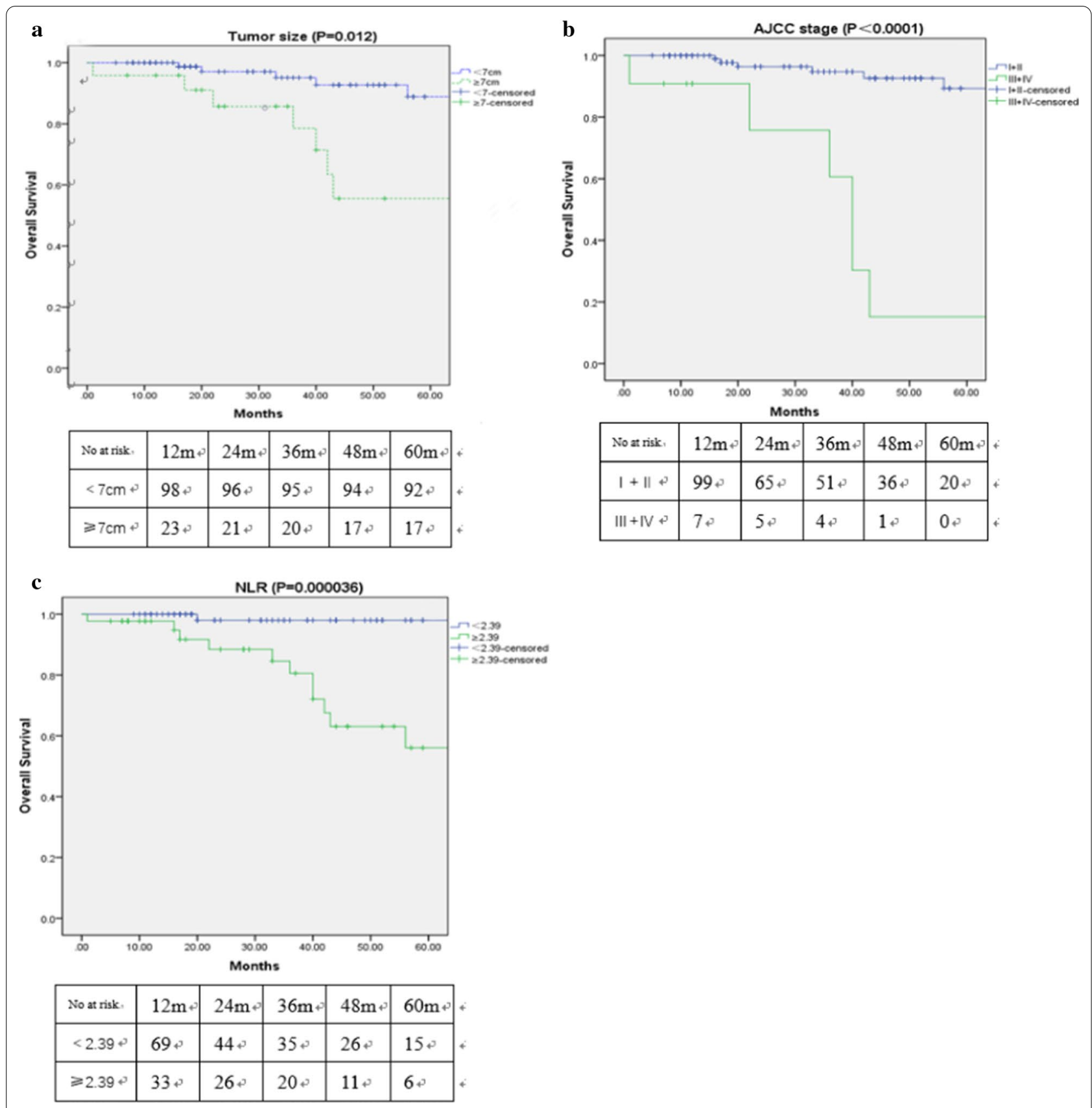

Fig. 1 Kaplan-Meier survival curves for OS of patients with PRCC (papillary renal cell carcinoma) according to tumor size (a), TNM (AJCC) stage (b) and NLR levels (c). Patients with larger tumor size, advanced TNM stage and higher NLR were associated with worse OS

more accurate in predicting overall survival than the TNM stage or NLR alone.

Several immunohistochemical biomarkers and nomograms have been confirmed to be independent prognostic factors for the survival of RCC patients $[16,17]$. The NLR is a tumor marker easily obtained from the peripheral blood. Previous research has reported that the NLR has been used to evaluate the tumor prognosis of localized renal cell carcinoma [18, 19], but most studies have focused on clear cell renal cell carcinoma, and there is a lack of studies on predictive models for pathological subtypes of papillary renal cell carcinoma. Huang et al. first reported that the NLR was associated with the RFS (recurrence-free survival) of non-metastatic papillary cell carcinoma patients [20], which revealed that an NLR $\geq 3.6$ was an independent predictor of RFS. This was similar to the conclusion of our study. Most different cutoff levels for the NLR with prognostic value for RCC range from 3 
Table 3 Univariable and multivariable analyses of factors in relation to overall survival

\begin{tabular}{|c|c|c|c|c|}
\hline \multirow[t]{2}{*}{ Variables } & \multicolumn{2}{|l|}{ Univariable } & \multicolumn{2}{|l|}{ Multivariable } \\
\hline & $\mathrm{HR}(95 \% \mathrm{Cl})$ & $P$ value & $\mathrm{HR}(95 \% \mathrm{Cl})$ & $P$ value \\
\hline \multicolumn{5}{|l|}{ Age/years } \\
\hline$\geq 60$ versus $<60$ & $1.072(0.376-3.063)$ & 0.896 & & \\
\hline \multicolumn{5}{|l|}{ Gender } \\
\hline$(\mathrm{M} \vee \mathrm{F} F)$ & $1.149(0.351-3.7571)$ & 0.818 & & \\
\hline \multicolumn{5}{|l|}{ BMl } \\
\hline$\geq 25$ versus $<25$ & $1.055(0.365-3.051)$ & 0.921 & & \\
\hline \multicolumn{5}{|c|}{ Diabetes/hyperglycemia, n (\%) } \\
\hline Yes versus no & $1.182(0.328-4.258)$ & 0.798 & & \\
\hline \multicolumn{5}{|l|}{ TNM stage (AJCC 8th) } \\
\hline$I I I+\mid V$ versus I+ II & $12.845(4.417-37.353)$ & 0.001 & $4.859(1.515-15.584)$ & 0.008 \\
\hline \multicolumn{5}{|l|}{ Surgical type } \\
\hline RN versus PN & $2.67(0.744-9.583)$ & 0.132 & & \\
\hline \multicolumn{5}{|l|}{ Tumor size (cm) } \\
\hline$\geq 7$ versus $<7$ & $3.557(1.24-10.205)$ & 0.018 & $1.819(0.60-5.51)$ & 0.290 \\
\hline \multicolumn{5}{|l|}{ Subtype, n (\%) } \\
\hline | versus || & $0.342(0.106-1.098)$ & 0.071 & & \\
\hline \multicolumn{5}{|l|}{ Serum album (g/l) } \\
\hline$<40$ versus $\geq 40$ & $0.641(0.214-1.92)$ & 0.427 & & \\
\hline \multicolumn{5}{|l|}{$\mathrm{CHOL}$} \\
\hline$<4.9$ versus $\geq 4.9$ & $0.988(0.344-2.838)$ & 0.983 & & \\
\hline \multicolumn{5}{|l|}{ NLR } \\
\hline$\geq 2.39$ versus $<2.39$ & $12.007(2.675-53.886)$ & 0.001 & $5.557(1.059-29.149)$ & 0.043 \\
\hline \multicolumn{5}{|l|}{$\mathrm{BUN} / \mathrm{Cr}$} \\
\hline$\geq 20$ versus $<20$ & $0.373(0.082-1.706)$ & 0.203 & & \\
\hline
\end{tabular}

TNM tumor-node-metastasis, $C H O L$ cholesterol, $N L R$ neutrophil-to-lymphocyte ratio, $P N$ partial nephrectomy, $R N$ radical nephrectomy

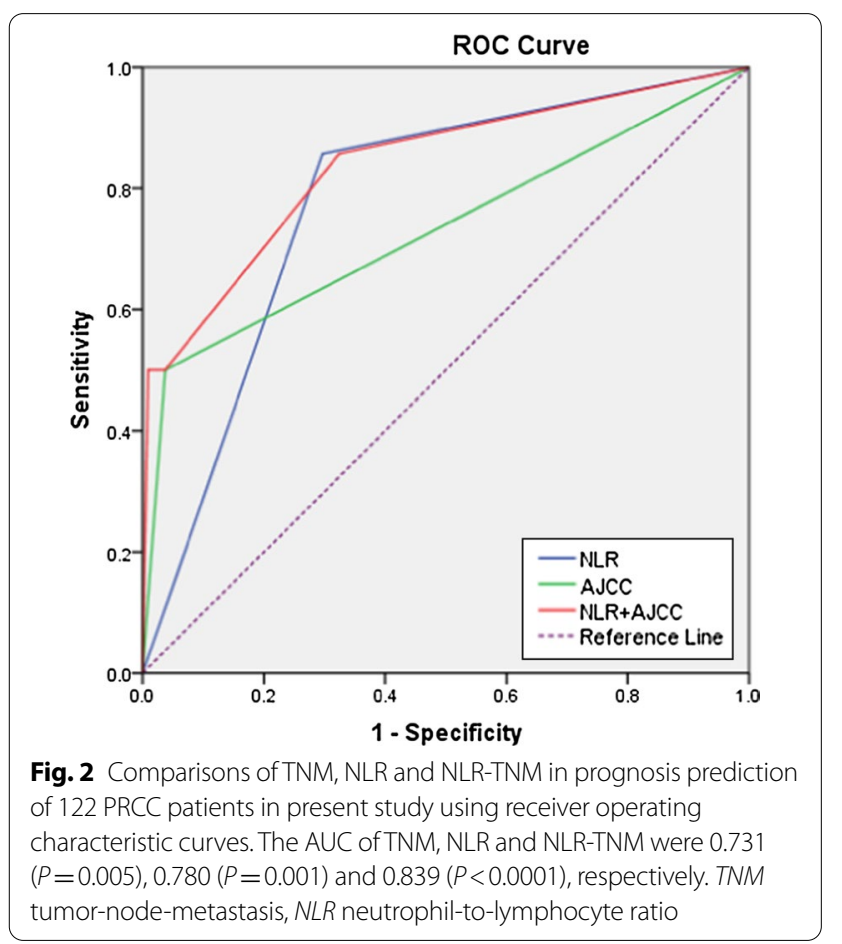

Table 4 Comparisons of the value of TNM, NLR and NLR-TNM systems in predicting prognosis of overall survival among the patients in present study

\begin{tabular}{lll}
\hline Model & AUC $(95 \% \mathrm{Cl})$ & $P$ \\
\hline TNM versus NLR-TNM & $\begin{array}{l}0.731(0.644-0.808) \text { versus } 0.839 \\
(0.761-0.899)\end{array}$ & 0.036 \\
NLR versus NLR-TNM $\begin{array}{l}0.780(0.696-0.850) \text { versus } 0.839 \\
(0.761-0.899)\end{array}$ & 0.034 \\
NLR versus TNM & $\begin{array}{l}0.780(0.696-0.850) \text { versus } 0.731 \\
(0.644-0.808)\end{array}$ & 0.489 \\
\hline
\end{tabular}

TNM tumor-node-metastasis, NLR neutrophil-to-lymphocyte ratio

to 5 [21], which was not consistent with our study, Most of the patients with PRCC in our study were staged early, and PRCC is a tumor with relatively low malignancy, which may contribute to this difference. Our study also found that a high preoperative NLR was related to poor overall survival in PRCC, indicating that the NLR could be used as an independent prognostic factor to predict prognosis.

The inflammatory response is an important factor in the occurrence of malignant tumors, and tumor cells 


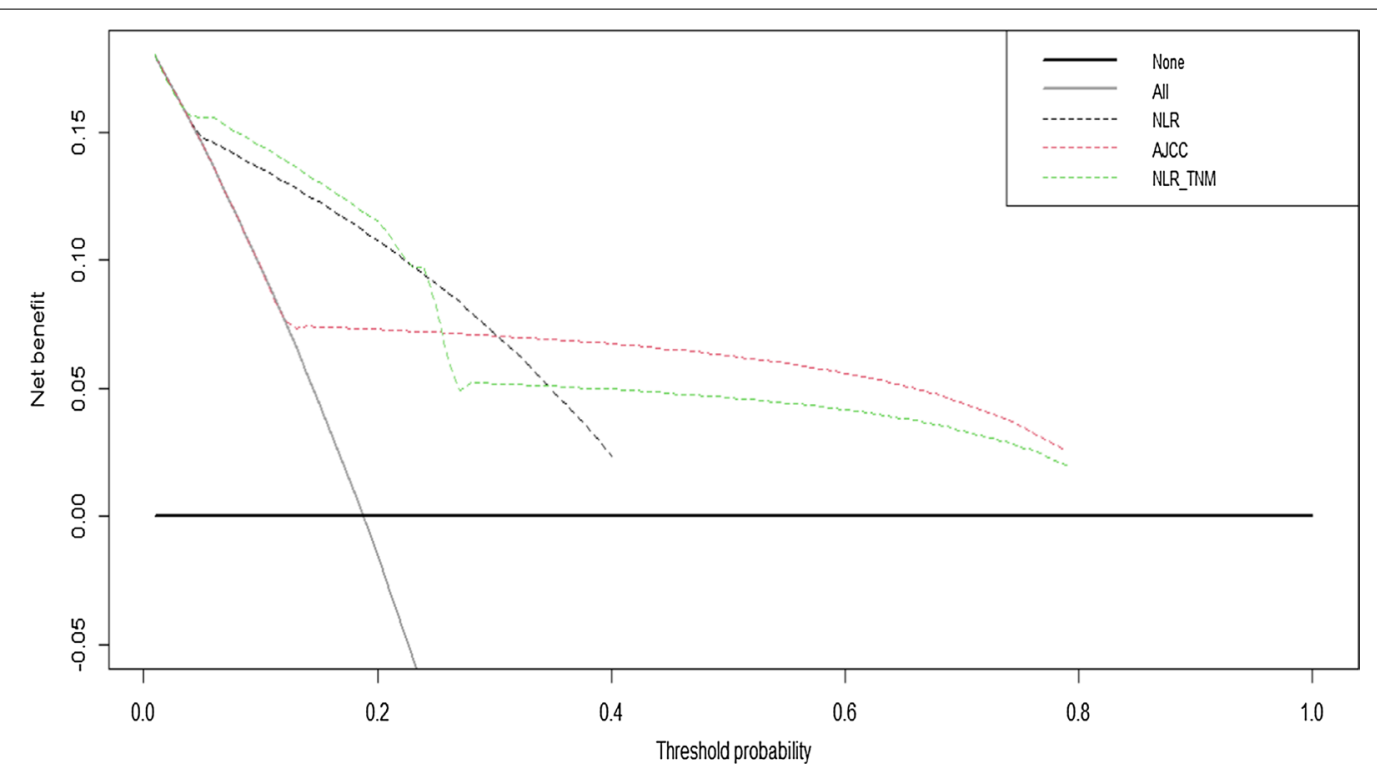

Fig. 3 Decision curve analysis for the TNM, NLR and NLR-TNM model. Notes: The $y$-axis measures the net benefit. The dotted line represents the model of TNM, NLR and NLR-TNM. The thin solid line represents the assumption that all patients are intervened. Thin thick solid line represents the assumption that no patients are intervened. The decision curve of NLR-TNM model showed the most benefit than the other two models

release a large number of inflammatory factors to change the tumor microenvironment, which can help tumor cells escape the host immune response. This escape leads to the proliferation and distant metastasis of tumor cells [22] and promotes the transformation of normal cells into tumor cells. Recent studies have shown that neutrophils can produce and release active cellular factors, such as IL6-1, IL-6 and VEGF, which changes the balance of inflammatory and anti-inflammatory in the tumor microenvironment [23]. Lymphocytes are important immune cells that contribute to tumor immune surveillance, which can inhibit tumor invasion. The NLR is a more accurate indicator of the inflammatory response level than either single factor composing the NLR. Therefore, a large number of studies have shown that a high NLR is associated with an advanced stage and a poor prognosis $[24,25]$, which was also supported by our study.

Since 2015, targeted therapy and immune checkpoint inhibitors have gradually become the main treatments for advanced metastatic renal carcinoma [26]. Vascular endothelial growth factor (VEGF) and vascular endothelial growth factor receptor (VEGFR) have become crucial targets of anticancer therapy because of their antiangiogenic functions. VEGF is a cellular activity factor produced by neutrophils, and whether the NLR can be used as a potential marker for the effect of targeted therapy remains to be determined. One study showed that the NLR was reduced by adjuvant sunitinib therapy for 4 weeks, with one endpoint of RFS improved [27]. Moreover, the NLR has been reported to be lower after the immunotherapy in metastatic RCC, with improved survival outcomes [28]. The systematic inflammatory response will be a very interesting focus of further research after neoadjuvant or adjuvant therapy in advanced RCC and PRCC.

Our study also had several limitations. First, our study was a respective study with a single-center, and the low incidence of PRCC led to the small sample of patients, so we did not divide the patients into metastatic PRCC and non-metastatic PRCC. Second, we did not evaluate the NLR after surgery, which limits the value for predicting prognosis. Third, due to a small number of disease specific deaths, we analyzed only OS as the primary endpoint.

\section{Conclusion}

This study showed that high preoperative NLR was associated with poor clinical and pathologic parameters of patients with PRCC. Moreover, NLR was also an independent prognostic factor of OS for patients with PRCC. The model of NLR-TNM integrating NLR with TNM stage could improve the ability of predicting overall survival.

\section{Abbreviations}

PRCC: Papillary renal cell carcinoma; NLR: Neutrophil-to-lymphocyte ratio; TNM: Tumor-node-metastasis; ROC: Receiver operating curve.

\section{Acknowledgements}

Not applicable. 


\section{Authors' contributions}

All authors have read and approved the manuscript. All authors have agreed both to be personally accountable for the own contributions and to ensure that questions related to the accuracy or integrity of any part of the work. ZZ: Data Collection, Manuscript writing; YY, JZ: Data collection: MZ and HN: made supervision and helped reviewing the manuscript. All authors read and approved the final manuscript.

\section{Funding}

No funding.

\section{Availability of data and materials}

Records and data pertaining to this study are in the patient's secure medical records in the Affiliated Hospital of Qingdao University, and the records and data could be accessed from ZZ.

\section{Ethics approval and consent to participate}

This study was approved by the Ethic Committee of the Affiliated Hospital of Qingdao University. Informed consent was obtained in both written and verbal format from patients or guardian for participants under 16 years old to participate, and the Ethic Committee of the Affiliated Hospital of Qingdao University approved the collection of verbal consent from participants.

\section{Consent for publication}

Not applicable.

\section{Competing interests}

Not applicable.

\section{Author details}

${ }^{1}$ Department of Urology, The Affiliated Hospital of Qingdao University, Qingdao, China. ${ }^{2}$ Department of Clinical Medicine, Qingdao University, Qingdao, China.

Received: 22 May 2020 Accepted: 2 March 2021

Published online: 22 March 2021

\section{References}

1. Bray F, Ferlay J, Soerjomataram I, Siegel RL, Torre LA, Jemal A. Global cancer statistics 2018: GLOBOCAN estimates of incidence and mortality worldwide for 36 cancers in 185 countries. CA Cancer J Clin. 2018;68(6):394-424.

2. Pichler M, Hutterer GC, Chromecki TF, et al. Renal cell carcinoma stage migration in a single European centre over 25 years: effects on 5- and 10-year metastasis-free survival. Int Urol Nephrol. 2012;44(4):997-1004.

3. Humphrey PA, Moch H, Cubilla AL, Ulbright TM, Reuter VE. The 2016 WHO classification of tumours of the urinary system and male genital organspart B: prostate and bladder tumours. Lyon: IARC Press; 2004.

4. Steffens $S$, Janssen M, Roos FC, et al. Incidence and long-term prognosis of papillary compared to clear cell renal cell carcinoma-a multicentre study. Eur J Cancer. 2012;48(15):2347-52.

5. Gores GJ. Inflammatory cytokines induce DNA damage and inhibit DNA repair in cholangiocarcinoma cells by a nitric oxide-dependent mechanism. Cancer Res. 2000;60(1):184-90.

6. Hainaut P, Plymoth A. Targeting the hallmarks of cancer: towards a rational approach to next-generation cancer therapy. Curr Opin Oncol. 2013;25(1):50.

7. Grebien F, Hantschel O, Wojcik J, et al. \& Weinberg, Robert A. Hallmarks of Cancer: The Next Generation. 2011.

8. Szkandera J, Stotz M, Absenger G, et al. Validation of C-reactive protein levels as a prognostic indicator for survival in a large cohort of pancreatic cancer patients. Br J Cancer. 2014;110(1):183-8.

9. Kazuya N, Kuniya T. The modified Glasgow prognostic score as a predictor of survival after hepatectomy for colorectal liver metastases. Ann Surg Oncol. 2014;21(5):1711-8.

10. Cho HB, Hur HW, Sang WK, et al. Pre-treatment neutrophil to lymphocyte ratio is elevated in epithelial ovarian cancer and predicts survival after treatment. Cancer Immunol Immunother. 2009;58(1):15-23.
11. Aliustaoglu M, Bilici A, Ustaalioglu BB, et al. The effect of peripheral blood values on prognosis of patients with locally advanced gastric cancer before treatment. Med Oncol. 2010;27(4):1060-5.

12. Templeton AJ, Ace O, Mcnamara MG, et al. Prognostic role of neutrophilto-lymphocyte ratio in solid tumors: a systematic review and meta-analysis. Breast Cancer Res. 2017;19(1):2.

13. Viers BR, Houston Thompson R, Boorjian SA, Lohse CM, Leibovich BC, Tollefson MK. Preoperative neutrophil-lymphocyte ratio predicts death among patients with localized clear cell renal carcinoma undergoing nephrectomy. Urol Oncol. 2014;32(8):1277-84.

14. Huang J, Dahl DM, Dong L, et al. Preoperative neutrophil-to-lymphocyte ratio and neutrophilia are independent predictors of recurrence in patients with localized papillary renal cell carcinoma. Biomed Res Int. 2015:2015:891045.

15. Wen RM, Zhang YJ, Ma S, Xu YL, Zheng JN. Preoperative neutrophil to lymphocyte ratio as a prognostic factor in patients with non-metastatic renal cell carcinoma. Asian Pac J Cancer Prev APJCP. 2015;16(9):3703-8.

16. Cindolo L, Patard J, Chiodini $P$, et al. Comparison of predictive accuracy of four prognostic models for nonmetastatic renal cell carcinoma after nephrectomy: a multicenter European study. Cancer. 2010;104(7):1362-71.

17. Karakiewicz PI, et al. Multi-institutional validation of a new renal cancerspecific survival nomogram. J Clin Oncol. 2007;25(11):1316-22.

18. Frank I, Blute ML, Cheville JC, Lohse CM, Weaver AL, Zincke H. An outcome prediction model for patients with clear cell renal cell carcinoma treated with radical nephrectomy based on tumor stage, size, grade and necrosis: the SSIGN score. J Urol. 2002;168(6):2395-400.

19. Viers BR, Boorjian SA, Frank I, et al. Pretreatment neutrophil-to-lymphocyte ratio is associated with advanced pathologic tumor stage and increased cancer-specific mortality among patients with urothelial carcinoma of the bladder undergoing radical cystectomy. Eur Urol. 2014;66(6):1157-64

20. Huang J, Dahl DM, Liang D, et al. Preoperative neutrophil-to-lymphocyte ratio and neutrophilia are independent predictors of recurrence in patients with localized papillary renal cell carcinoma. Biomed Res Int. 2015:2015:1-9.

21. Byun SS, Hwang EC, Kang SH, et al. Prognostic significance of preoperative neutrophil-to-lymphocyte ratio in nonmetastatic renal cell carcinoma: a large. Multicenter Cohort Analysis Biomed Res Int. 2016:2016:5634148.

22. Byong S, Oh J, Won J. Prognostic value of C-reactive protein and neutrophil-to-lymphocyte ratio in patients with hepatocellular carcinoma. BMC Cancer. 2013;13:78

23. Ingrid M, Markus M, Pascale K, Maria HG. Polymorphonuclear neutrophils and T lymphocytes: strange bedfellows or brothers in arms. Trends Immu nol. 2009:30(11):522-30.

24. Hsu JT, Liao CK, Le PH, et al. Prognostic value of the preoperative neutrophil to lymphocyte ratio in resectable gastric cancer. Medicine (Abingdon). 2015;94(39):e1589.

25. Gu XB, Tian T, Tian XJ, Zhang XJ. Prognostic significance of neutrophil-tolymphocyte ratio in non-small cell lung cancer: a meta-analysis. Sci Rep. 2015;5:12493.

26. Soerensen AV, Donskov F, Hermann GG, et al. Improved overall survival after implementation of targeted therapy for patients with metastatic renal cell carcinoma: results from the Danish Renal Cancer Group (DARENCA) study-2. Eur J Cancer. 2014;50(3):553-62.

27. Patel A, Ravaud A, Motzer RJ, Pantuck AJ, George DJ. Neutrophil-tolymphocyte ratio as a potential prognostic factor of disease-free survival in high-risk renal cell carcinoma: analysis of the S-TRAC trial. J Clin Oncol. 2018;36(15_suppl):4562-4562.

28. Lalani AA, Xie W, Martini DJ, et al. Change in Neutrophil-to-lymphocyte ratio (NLR) in response to immune checkpoint blockade for metastatic renal cell carcinoma. J Immunother Cancer. 2018;6(1):5.

\section{Publisher's Note}

Springer Nature remains neutral with regard to jurisdictional claims in published maps and institutional affiliations. 